ESAIM: PROCEEDINGS AND SURVEYS, September 2014, Vol. 45, p. 493-501

J.-S. Dhersin, Editor

\title{
SCALAR CONSERVATION LAW WITH DISCONTINUITY ARISING IN PEDESTRIAN MODELING*
}

\author{
Matthias Mimault $^{1}$
}

\begin{abstract}
We consider a generalized version of the Hughes' macroscopic model of pedestrian motion. It consists of a conservation law on the pedestrian mass with an eikonal equation giving the direction of the flux depending of the density. The model displays a non-classical dynamics at the splitting point. Known convergence results for finite volume schemes do not apply in this setting. The wavefront tracking provides us with reference solutions to test numerically the convergence of classical finite volume schemes. These schemes will be used with a tracking algorithm to show the path of a single pedestrian during an evacuation.

Résumé. On considère une version généralisée du modèle macroscopique de mouvements de foule de Hughes. Il se compose d'une loi de conservation sur la masse de piétons couplée à une équation eikonale donnant la direction du flux selon la répartition des densités. Ce modèle montre une dynamique nonclassique au niveau du point de césure. Les résultats de convergence pour les schémas aux volumes finis ne s'appliquent pas dans cette configuration. La méthode de suivi des fronts fournit une solution de référence pour tester numériquement la convergence de schémas classiques de volumes finis. Ces schémas seront utilisés pour tracer la trajectoire d'un piéton unique à l'aide d'un algorithme additionnel.
\end{abstract}

\section{INTRODUCTION}

The understanding of pedestrian behavior is crucial for crowd management. Effects of density take over the individual behavior and reactions to panicking situation can be hazardous. Hughes [6] proposed a macroscopic model of the pedestrian flow : the pedestrian density $\rho=\rho(t, x)$ evolves according to a scalar conservation law, where the preferred direction of motion is given by the solution of an eikonal equation.

This paper addresses the numerical simulation of the evacuation of a one-dimensional corridor with two exits. The crowd will split in two parts but the separation point is not a priori fixed. The one dimensional model reads as

$$
\begin{gathered}
\rho_{t}-\left(\rho v(\rho) \frac{\phi_{x}}{\left|\phi_{x}\right|}\right)_{x}=0, \\
\left|\phi_{x}\right|=c(\rho),
\end{gathered}
$$

in the spatial domain $\Omega=]-1,1[$. The above equations must be completed with homogeneous Dirichlet boundary conditions at $x= \pm 1$, which represent the exit locations

* This research was supported by the European Research Council under the European Union's Seventh Framework Program (FP/2007-2013) / ERC Grant Agreement n. 257661.

${ }^{1}$ INRIA Sophia-Antipolis; e-mail: matthias.mimault@inria.fr

(C) EDP Sciences, SMAI 2014 


$$
\begin{aligned}
& \rho(t,-1)=\rho(t, 1)=0, \\
& \phi(t,-1)=\phi(t, 1)=0,
\end{aligned}
$$

and an initial density profile $\rho(0,)=.\rho_{0} \in \mathrm{BV}(\mathbb{R}) . x \in \Omega$ is the space variable, $t \in \mathbb{R}^{+}$is the time, $\rho(t, x) \in[0,1]$ is the normalized crowd density, $c=c(\rho)$ is the cost function, $v(\rho)=1-\rho$ is the mean velocity and we set $f(\rho)=\rho v(\rho)=\rho(1-\rho)$. We assume that $c:[0,1[\rightarrow[1,+\infty[$ is a smooth function such that $c(0)=1$ and $c^{\prime}(\rho) \geq 0$ for $\rho \in[0,1[$. Recall that the literature usually proposes the choice

$$
c(\rho)=\frac{1}{v(\rho)}
$$

as in $[3,6,7]$.

We observe that (1) can be rewritten as

$$
\rho_{t}-\left(f(\rho) \operatorname{sgn}\left(\phi_{x}\right)\right)_{x}=0,
$$

and the unique viscosity solution to the Dirichlet problem $(2),(4)$ is given by the value function of the corresponding control problem with discontinuous coefficient $c(\rho)$, i.e.

$$
\phi(t, x)=\left\{\begin{array}{l}
\int_{-1}^{x} c(\rho(t, y)) d y \text { if } x \leq \xi(t) \\
\int_{x}^{1} c(\rho(t, y)) d y \text { if } x \geq \xi(t)
\end{array}\right.
$$

where $\xi(t)$ is implicitly defined by the identity

$$
\int_{-1}^{\xi(t)} c(\rho(t, y)) d y=\int_{\xi(t)}^{1} c(\rho(t, y)) d y .
$$
flux :

Therefore, equation (1) can be written as a scalar conservation law with discontinuous space-time dependent

$$
\rho_{t}+F(t, x, \rho)_{x}=0
$$

where $F(t, x, \rho)=\operatorname{sgn}(x-\xi(t)) f(\rho)$. As the position of the discontinuity $\xi$ is not fixed and depends nonlocally on $\rho$ itself, known convergence results cannot be applied in this configuration. Nevertheless, evidences of numerical convergence of finite volume schemes has been produced using accurate approximations of entropy weak solutions computed using the wave-front algorithm detailed in [4]. It captures by construction the exact behavior of the solution at the turning point using the Theorem 1 of [1] and gives a reference solution used to appreciate the convergence of the finite volume scheme.

In Section 1, we present a wave-front tracking method, defining directly the nature of the solution at the turning point. Section 2 shows the approximation of the solution with finite volume methods, without any specific attention to the treatment of the separation point. In the last section, we complete the finite volume methods with the tracking of a single pedestrian, using the algorithm developed by Bretti and Piccoli [2]. The 

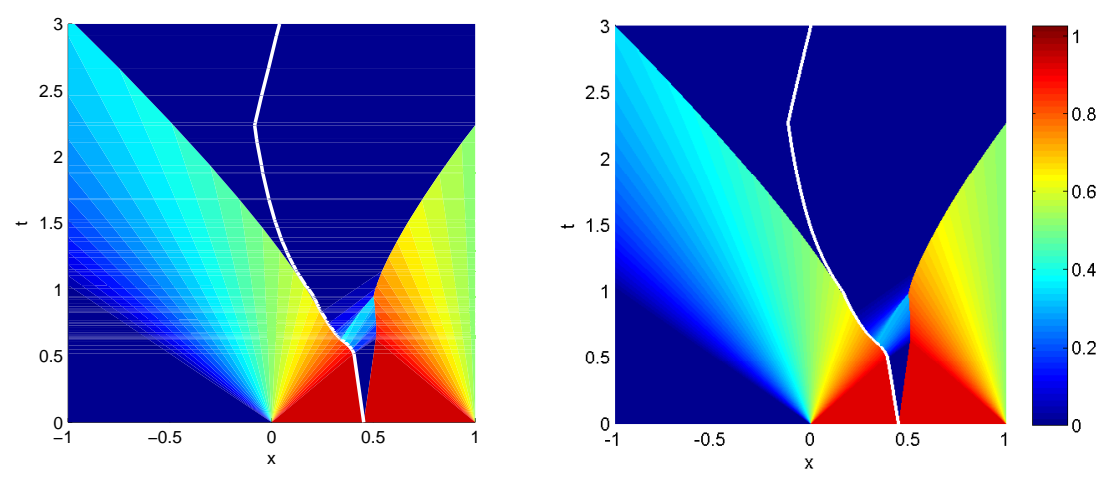

FiguRE 1. Wave-front tracking solution of (11) up to time $T=3$ with $\rho_{0}(x)=0$ for $\left.x \in\right]-1,0[$ and $\rho_{0}(x)=0.9$ for $\left.x \in\right] 0,1\left[\right.$, and mesh sizes $\Delta \rho=2^{-4}$ (left) and $\Delta \rho=2^{-10}$ (right). The white curve is the trajectory $x=\xi(t)$.
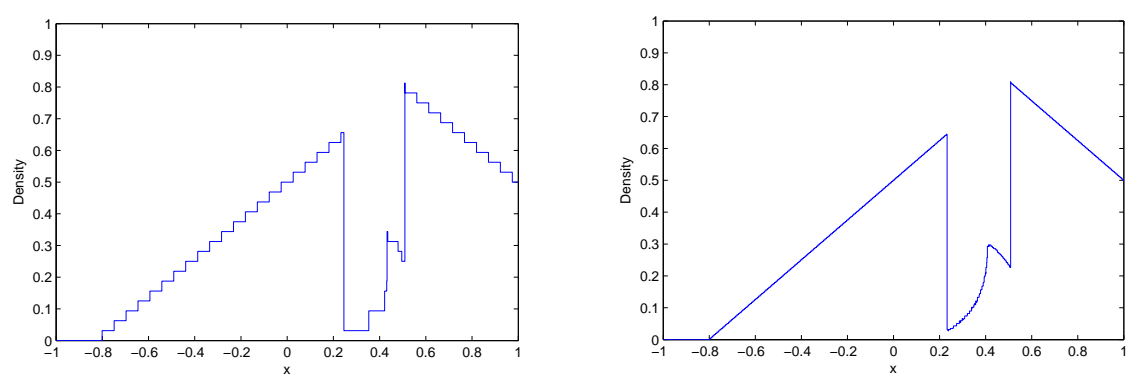

Figure 2. Density profiles at time $T=0.8$ with initial density $\rho_{0}(x)=0$ for $\left.x \in\right]-1,0[$ and $\rho_{0}(x)=0.9$ for $\left.x \in\right] 0,1[$, computed using wave-front tracking scheme with mesh size $\Delta \rho=2^{-4}$ (left) and $\Delta \rho=2^{-10}$ (right). The position of the turning point coincides with the first downward jump discontinuity from the left.

plot of the trajectory displays the change of direction and gives a better insight on the turning phenomena which can happen during a simulation.

\section{Approximate solutions}

\subsection{Wave-front tracking}

The wave-front tracking method constructs a piecewise constant exact solution of an approximated problem with a piecewise constant initial datum and piecewise linear flux $f^{\nu}$. It coincides with $f$ on the mesh $\mathcal{M}_{\nu}=$ $\left\{\rho_{i}^{\nu}\right\}_{i=0}^{2^{\nu}} \subset \Omega$ defined by

$$
\mathcal{M}_{\nu}=\left(2^{-\nu} \mathbb{N} \cap[0,1]\right)
$$

for $\nu \in \mathbb{N}, \nu>0$.

For small times $t>0$, a piecewise approximate solution $\left(\rho^{\nu}, \xi_{\nu}\right)$ to problem $(1),(2)$ is constructed piecing together the solutions to the local Riemann problems 


$$
\left\{\begin{array} { l } 
{ \partial _ { t } \rho + \partial _ { x } ( \operatorname { s g n } ( x - \xi _ { 0 } ) f ^ { \nu } ( \rho ) ) = 0 , } \\
{ \rho ( 0 , x ) = \{ \begin{array} { l } 
{ \rho _ { 0 , 0 } ^ { \nu } \text { if } x < \xi _ { 0 } , } \\
{ \rho _ { 0 , 1 } ^ { \nu } \text { if } x > \xi _ { 0 } , } \\
{ \dot { \xi } ( \rho _ { + } - \rho _ { - } ) = \Psi [ \rho ] , }
\end{array} }
\end{array} \left\{\begin{array}{l}
\partial_{t} \rho+\partial_{x}\left(\operatorname{sgn}\left(x_{j}-\xi_{0}\right) f^{\nu}(\rho)\right)=0, \\
\rho(0, x)=\left\{\begin{array}{c}
\rho_{0, j}^{\nu} \text { if } x<x_{j}, \\
\rho_{0, j+1}^{\nu} \text { if } x>x_{j},
\end{array}\right. \\
j \neq 0 .
\end{array}\right.\right.
$$

Solving the Riemann problems above replaces smooth rarefaction fronts by rarefaction fans of constant values $\rho_{j}^{\nu}, j=l_{1}, \ldots, l_{N_{\nu}}$, such that $\left|\rho_{j}^{\nu}-\rho_{j-1}^{\nu}\right|=2^{-\nu}$, separated by jump discontinuities moving with speeds $\lambda_{j}=1-\rho_{j}^{\nu}-\rho_{j-1}^{\nu}$, see Fig. 1, left. Note that the solution to the Riemann problem in (11), left, is constructed by means of the Riemann solver described in [4] where here is shown an extract in the case $\rho_{L}<\rho_{R}$ :

a) If

$$
\Psi *<\frac{f\left(\rho_{R}\right)+f\left(\rho_{L}\right)}{\rho_{R}-\rho_{L}}\left[c\left(\rho_{R}\right)+c\left(\rho_{L}\right)\right]
$$

then there exists a unique intermediate value $\rho_{M}$, with $\rho_{L}<\rho_{M}<\rho_{R}$, such that the solution is given by the turning curve $\xi$ followed by a rarefaction between $\rho_{M}$ and $\rho_{R}$.

b) If

$$
\frac{f\left(\rho_{R}\right)+f\left(\rho_{L}\right)}{\rho_{R}-\rho_{L}}\left[c\left(\rho_{R}\right)+c\left(\rho_{L}\right)\right] \leq \Psi * \leq-v\left(\rho_{L}\right)\left(1+c\left(\rho_{L}\right)\right)-v\left(\rho_{R}\right)\left(1-c\left(\rho_{R}\right)\right),
$$

then a unique intermediate value $\rho_{M} \in\left[0, \rho_{R}\right]$ exists, such that the solution is given by the turning curve $\xi$ followed by a shock between $\rho_{M}$ and $\rho_{R}$. If the equality holds in the r.h.s. of (13), then $\rho_{M}=0$ : vacuum appears between the turning point and the shock.

c) If

$$
-v\left(\rho_{L}\right)\left(1+c\left(\rho_{L}\right)\right)-v\left(\rho_{R}\right)\left(1-c\left(\rho_{R}\right)\right)<\Psi *<v\left(\rho_{R}\right)\left(1+c\left(\rho_{R}\right)\right)+v\left(\rho_{L}\right)\left(1-c\left(\rho_{L}\right)\right),
$$

then the solution is given by a shock of speed $\rho_{L}-1$ followed by $\xi$ and by a shock of speed $1-\rho_{R}$, the intermediate state around $\xi$ being $\rho_{M}=0$.

d) Finally if

$$
\Psi * \geq v\left(\rho_{R}\right)\left(1+c\left(\rho_{R}\right)\right)+v\left(\rho_{L}\right)\left(1-c\left(\rho_{L}\right)\right)
$$

then the solution is given by a shock between $\rho_{L}$ and $\rho_{M} \in\left[0, \rho_{R}\right]$ followed by $\xi$. If the equality holds in (15), then $\rho_{M}=0$, otherwise $\rho_{M}>0$.

Recall that $\Psi^{*}:=\left\{\int_{\xi+\delta}^{1}-\int_{-1}^{\xi-\delta}\right\} \partial_{t}[c(\rho(t, y))] d y$, is the relative evacuation rate of the corridor and it is supposed to be constant for small times and $\rho_{L}$ and $\rho_{R}$ are left and right states around $\xi_{0}$.

Once the density values on both sides of the turning curve have been computed, the speed of the approximated turning curve $\dot{\xi}_{\nu}$ is evaluated using the Rankine-Hugoniot equation in (11), left. The trajectory of the turning point is updated

$$
\xi_{\nu}(t)=\xi_{0}+\dot{\xi}_{\nu} t
$$

The new density values $\rho_{1, j_{1}}^{\nu}, \ldots, \rho_{1, j_{N_{\nu}}}^{\nu}$ resulting from the solution of each Riemann problem are interposed to the corresponding density values $\rho_{0, j}^{\nu}, \rho_{0, j+1}^{\nu}$, and all are relabelled $\rho_{1, j}^{\nu}, j \in \mathbb{Z}$. To each couple of subsequent 
density values is associated the speed of the corresponding wave $\lambda_{1, j}$ or $\dot{\xi}_{\nu}$ and the corresponding departure point $x_{j}$. This allows to compute the corresponding wave trajectories $x_{j}(t)=x_{j}+\lambda_{1, j} t\left(\right.$ respectively $x_{0}(t)=$ $\xi_{\nu}(t)=\xi_{0}+\dot{\xi}_{\nu} t$ for the turning curve) and to update the solution

$$
\rho^{\nu}(t, .)=\sum_{j \in \mathbb{Z}} \rho_{1, j}^{\nu} \chi_{\left[x_{j-1}(t), x_{j}(t)\right]},
$$

for small $t>0$.

The piecewise constant approximate solution $\rho^{\nu}$ constructed above can be prolonged up to the first time $\bar{t}>0$, where two discontinuities collide, or a discontinuity hits the turning curve $\xi^{\nu}$. In both cases, a new Riemann problem arises and its solution, obtained in the former case with the classical Riemann solver and in the latter by means of the particular one described in [4], allows to extend $\rho^{\nu}, \xi^{\nu}$ further in time. We point out that the absorbing boundary conditions (3) are taken into account by simply dropping the waves hitting the left and right boundaries.

\subsection{Finite volume schemes}

Here we aim at investigating the behavior of classical numerical schemes near the turning curve, and whether they manage to capture correctly the behavior of the solution constructed via the wave-front tracking algorithm.

Given an initial datum $\rho(0, x)=\rho_{0}(x)$ and homogeneous Dirichlet boundary conditions $(3),(4)$, we solve (1),(2) in an iterative manner at each time step, i.e.

(1) Given $\rho$, solve the eikonal equation (2) by the fast sweeping method [9].

(2) Given $\phi$, solve the non-linear conservation law (1) using Godunov scheme [5].

The domain $[-1,1]$ is divided into $N$ uniform cells $I_{j}=\left[x_{j-1 / 2}, x_{j+1 / 2}\right]$ with centers at points $x_{j}=j \Delta x$, with $\Delta x=2 / N$.

The explicit algorithm used to generate the approximations $\rho_{j}^{n}$ was introduced by Towers in [8] and is written in conservation form

$$
\rho_{j}^{n+1}=\rho_{j}^{n}-\frac{\Delta t^{n}}{\Delta x}\left(k_{j+\frac{1}{2}}^{n} \mathrm{~h}_{j+\frac{1}{2}}^{n}-k_{j-\frac{1}{2}}^{n} \mathrm{~h}_{j-\frac{1}{2}}^{n}\right)
$$

where $\Delta t^{n}=t^{n+1}-t^{n}$ is chosen to satisfy the following CFL condition

$$
\Delta t^{n}<0.5 \frac{\Delta x}{\max \left\{\max _{j}\left|f^{\prime}\left(\rho_{j}^{n}\right)\right|,\left|\dot{\xi}^{n}\right|\right\}} .
$$

In the above formula, the coefficient 0.5 is chosen to avoid interactions of $\xi$ with the cell boundaries and $|\dot{\xi}|$ is estimated at each time step by differentiating the implicit expression (8). As its role is to direct the fluxes, $\xi$ 's new position is assumed at the center of its cell.

In (18), we set $k_{j \pm 1 / 2}^{n}=\operatorname{sgn}\left(\phi_{x}^{n}\left(x_{j \pm 1 / 2}\right)\right)$, where

$$
\phi_{x}^{n}\left(x_{j+1 / 2}\right) \simeq \frac{\phi^{n}\left(x_{j+1}\right)-\phi^{n}\left(x_{j}\right)}{\Delta x} .
$$

The numerical flux $\mathrm{h}_{j+1 / 2}^{n}=h\left(\rho_{j}^{n}, \rho_{j+1}^{n}\right)$ is chosen to be monotone and consistent, i.e. $h(\rho, \rho)=f(\rho)=\rho v(\rho)$. In order to maintain the monotonicity of the scheme, we transpose the arguments when $k_{j+1 / 2}$ changes sign, i.e. 


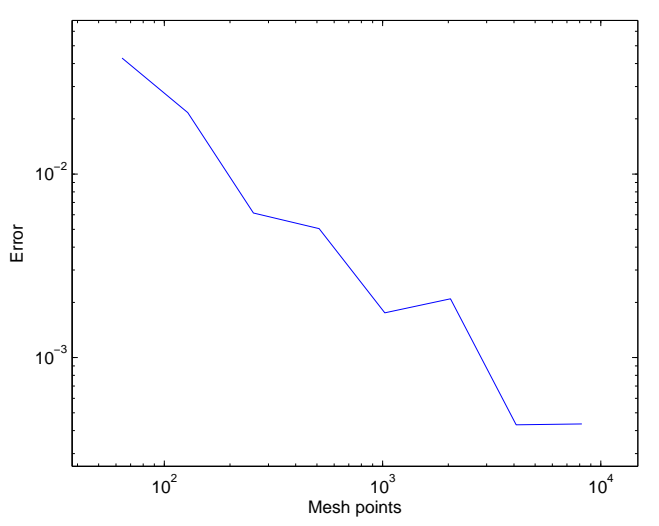

Figure 3. $\mathbf{L}_{1}$-error $\epsilon_{\nu}$ between two subsequent discretization meshes $2^{-\nu}$ and $2^{-\nu-1}$, for $\nu=$ $5, \ldots, 12$, corresponding to the initial datum given by $(22)$.

$$
\mathrm{h}_{j+\frac{1}{2}}= \begin{cases}h\left(\rho_{j}, \rho_{j+1}\right) & \text { if } k_{j+\frac{1}{2}} \geq 0 \\ h\left(\rho_{j+1}, \rho_{j}\right) & \text { if } k_{j+\frac{1}{2}}<0 .\end{cases}
$$

with $h$ depending of the chosen flux.

\section{Results}

In this section we provide some numerical tests showing the convergence of the wave-front tracking and finite volumes schemes described in previous sections. To this end, we choose an initial datum $\rho_{0}$ that gives a non-trivial behavior at the turning curve

$$
\rho_{0}(x)= \begin{cases}0 & \text { if }-1<x<0, \\ 0.9 & \text { if } 0<x<1,\end{cases}
$$

and the cost function $c(\rho)=1 / v(\rho)=1 /(1-\rho)$. We run simulations up to time $T=3$, so that the whole mass leaves the domain $\Omega$ and $\rho(T, x) \equiv 0$, see Fig. 1 .

\subsection{Reference solution}

Wave-front tracking solutions are not defined on a standard cartesian grid in space and time. In order to compare the approximate solutions obtained via wave-front tracking at different mesh sizes and finite volume approximations, we have to convert these data sets to values defined on a reference cartesian grid using the method described in [4]. We call $\tilde{\rho}_{\mathrm{WFT}}^{\nu}$ values obtained by taking the corresponding values of $\rho_{\mathrm{WFT}}^{\nu}$ at the cartesian grid nodes. Finally, we operate a $\mathbf{L}_{1}-$ norm comparison between two subsequent discretization meshes $2^{-\nu}$ and $2^{-\nu-1}$ according to the following formula

$$
\epsilon_{\nu}=\left\|\tilde{\rho}_{W F T}^{\nu+1}-\tilde{\rho}_{W F T}^{\nu}\right\|_{\mathbf{L}_{1}}
$$

which decreases to zero as $\nu$ increases. Fig. 3 shows numerically that the sequence $\left\{\tilde{\rho}_{W F T}^{\nu}\right\}$ is a Cauchy sequence in $\mathbf{L}_{1}$. 


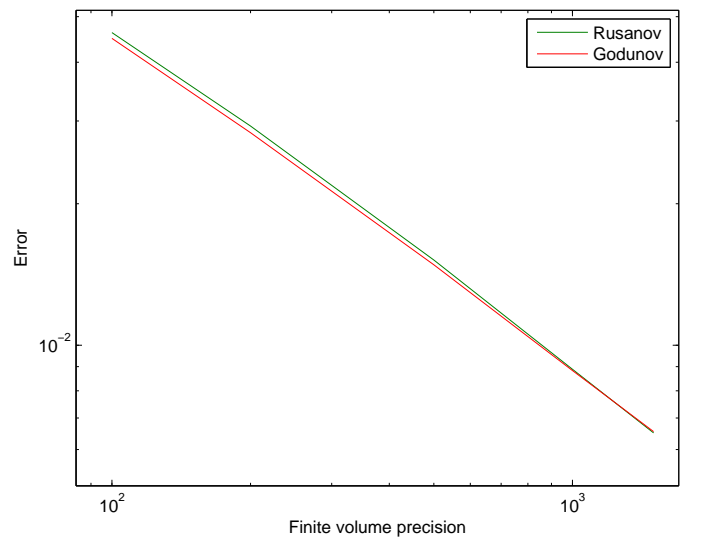

FiguRE 4. $\mathbf{L}_{1}$-norm of the error in logarithmic scale for mesh sizes $\Delta x=1 / 50, \ldots, 1 / 1500$, for Godunov schemes corresponding to the initial datum given by (22). The convergence of Rusanov scheme is also shown for illustration.

\subsection{Convergence of finite volumes}

Once assessed the convergence of the wave-front tracking scheme, we take as reference "exact" solution the one obtained with density mesh size $\varepsilon=2^{-10}$, and we compare it to the approximations computed by Godunov scheme for different space mesh sizes, computing the $\mathbf{L}_{1}$-norm of the difference.

Fig. 4 reports the values of the $\mathbf{L}_{1}$-error computed on the time interval $[0, T]$ with $T=1.2$, in order to focus on the non-classical behavior of the solution. Indeed, at $T=1.2$ vacuum has appeared around the turning point $\xi$, and the solution has become classical. For a set of space meshes ranging from $\Delta x=1 / 50$ to $\Delta x=1 / 1500$, we see a clear linear decreasing in logarithmic scale, showing numerically a convergence order of about 0.67 .

\section{The SINGLE PEDESTRIAN'S TRAJECTORY}

In paper [2], Bretti and Piccoli build the trajectory of a single vehicle in traffic flow. This algorithm will be extended to our pedestrian simulation with a discontinuous flux function. Assuming that the pedestrian moves with an average speed $v=v(\rho)$, its trajectory will be the solution of the ordinary differential equation

$$
\left\{\begin{array}{l}
\dot{x}(t)=v(\rho(t, x(t))), \\
x(\bar{t})=\bar{x} .
\end{array}\right.
$$

where $x$ is the position of the particle at time $t$. Its speed depends on the density $\rho$ but it does not affect the density distribution, so the ODE will be weakly coupled to the rest of the equations.

\subsection{Algorithm}

The model (1), (2) has a bidirectional flux discontinuous in $x=\xi(t)$. The computation of the pedestrian's trajectory with a convex flux will be symmetric with respect to the concave part so we will explain it in stream terms. We define

- $x_{p}$ the position of the particle at the beginning of the time step,

- $m$ the index of the cell $C_{m}$ containing $x_{p}$,

- $x_{m}$ the starting point of the wave following $x_{p}$, 

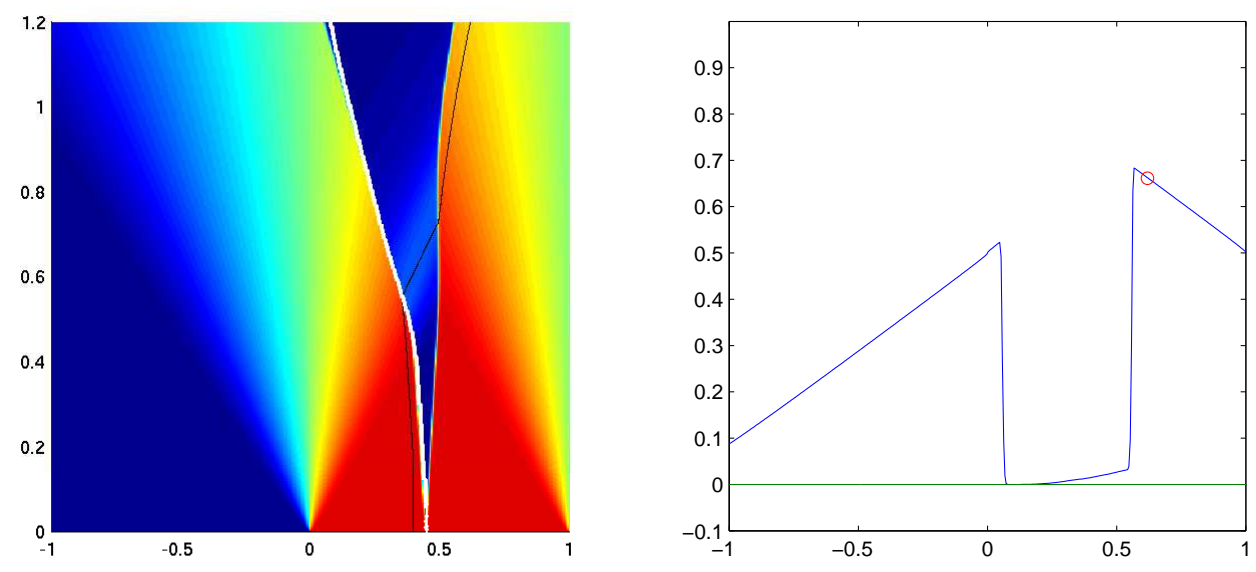

FiguRE 5. Trajectory in black during the evolution of 22 and its position in red circle on the density profile of a single pedestrian starting at $x_{0}=0.4$. The simulation parameters are $\rho_{0}(x)=0$ for $\left.x \in\right]-1,0\left[\right.$ and $\rho_{0}(x)=0.9$ for $\left.x \in\right] 0,1[, t=1.2$ on right and $\Delta x=0.005$.

- $\rho_{1}$ the density in $C_{m}$,

- $\rho_{2}$ the density in the downstream cell.

At each time step we compute the new position of the pedestrian. On either side of the turning point, the flow is monotone, so that a particle can interact only with the downstream waves.

To check if the pedestrian interacts with the wave during the time step, we compute $\bar{t}=\frac{x_{m}-x_{p}}{v\left(\rho_{1}\right)-\lambda_{m}}$, where $\lambda_{m}=\frac{f\left(\rho_{2}\right)-f\left(\rho_{1}\right)}{\rho_{2}-\rho_{1}}$, is computed. $\bar{t}$ represents the time when the particle interacts with the next wave. $\lambda_{m}$ is the speed of this wave and we distinguish two cases :

- if $\bar{t}>\Delta t$ the particle does not cross the wave. Its new position is $x_{p}^{*}=x_{p}+v\left(\rho_{1}\right) \Delta t$,

- if $\bar{t}<\Delta t$ we discern two additional cases :

- if $\rho_{1}<\rho_{2}$ the wave is a shock and $x_{p}^{*}=x_{p}+\bar{t} v\left(\rho_{1}\right)+(\Delta t-\bar{t}) v\left(\rho_{2}\right)$,

- if $\rho_{>} \rho_{2}$ the pedestrian interacts with a rarefaction. We have to define if at the end of the time step, the pedestrian has crossed it or it is still inside it. We define $\overline{\bar{t}}$ the time when the pedestrian exits the rarefaction wave. It is defined implicitly by

$$
\frac{x(\overline{\bar{t}})-x_{m}}{\overline{\bar{t}}}=f^{\prime}\left(\rho_{2}\right),
$$

where $x(t)=x_{m}+\left(t-t_{0}\right)-\bar{t} \frac{\sqrt{\left(t-t_{0}\right)}}{\sqrt{(} \bar{t})}\left(1-v\left(\rho_{1}\right)\right)$ and $t_{0}$ the global time at the beginning of the time step.

The computation of the trajectory is automatic at $\xi$ because, by assumption, it is at the center of the corresponding cell. What matters in computation of $x_{p}$ 's trajectory is the flux direction at cell's boundaries. We do not compute explicitly a direct contact with $\xi$ because it is always upstream with respect to $x_{p}$. In case of equality, $x_{p}$ will be counted on the right side of $\xi$ for convenience reasons.

\subsection{Results}

Being able to follow the path of a single pedestrian reveals clearly the change of direction operated by a person choosing a different exit to evacuate. The choice is made according to the evolution of the density profile 
during the evacuation. The evacuation at the doors makes the density decrease but the spreading of pedestrians along the corridor increases dramatically the relative cost of a travel to join the first chosen exit. On Fig. 5, the trajectory and the location of a pedestrian is depicted. First he chooses to join the left exit but he takes another direction when the balance of the costs changes in favor of the right one.

\section{Conclusion}

The evacuation of pedestrians along a narrow corridor results in a problem with a conservation law, where the position of the turning point depends on the densities distribution on the whole domain. The wave-front tracking method was coded to include the behavior of the solution at the turning point. We prove numerically that classical Godunov scheme has a good convergence. Thus the tracking algorithm works well with the discontinuity even though there is no explicit treatment of it.

\section{REFERENCES}

[1] Debora Amadori and M. Di Francesco. The one-dimensional Hughes model for pedestrian flow: Riemann-type solutions. Acta Math. Sci. Ser. B Engl. Ed., 32(1):259-280, 2012.

[2] Gabriella Bretti and Benedetto Piccoli. A tracking algorithm for car paths on road networks. SIAM J. Appl. Dyn. Syst., $7(2): 510-531,2008$.

[3] Marco Di Francesco, Peter A. Markowich, Jan-Frederik Pietschmann, and Marie-Therese Wolfram. On the Hughes' model for pedestrian flow: the one-dimensional case. J. Differential Equations, 250(3):1334-1362, 2011.

[4] Paola Goatin and Matthias Mimault. The wave-front tracking algorithm for Hughes' model of pedestrian motion. SIAM J. Sci. Comput., 35(3):B606-B622, 2013.

[5] S. K. Godunov. A difference method for numerical calculation of discontinuous solutions of the equations of hydrodynamics. Mat. Sb. (N.S.), 47 (89):271-306, 1959.

[6] Roger L. Hughes. A continuum theory for the flow of pedestrians. Transpn. Res. - B, 36(6):507-535, 2002.

[7] Roger L. Hughes. The flow of human crowds. In Annual review of fluid mechanics, Vol. 35, volume 35 of Annu. Rev. Fluid Mech., pages 169-182. Annual Reviews, Palo Alto, CA, 2003.

[8] John D. Towers. Convergence of a difference scheme for conservation laws with a discontinuous flux. J. Numer. Anal., 38(2):681$698,2000$.

[9] Hongkai Zhao. A fast sweeping method for eikonal equations. Math. Comp., 74(250):603-627 (electronic), 2005. 